\title{
Consumer perceptions of direct-to-consumer personalized genomic risk assessments
}

\author{
Cinnamon S. Bloss, PhD $D^{1}$, Laura Ornowski, $M S^{1,2}$, Elana Silver, $M S^{3}$, Michele Cargill, PhD ${ }^{3}$, \\ Vance Vanier, $M D^{3}$, Nicholas J. Schork, PhD ${ }^{1,4}$, and Eric J. Topol, MD ${ }^{1,2,4}$
}

\begin{abstract}
Purpose: To evaluate consumer perceptions of direct-to-consumer personalized genomic risk assessments and assess the extent to which consumer characteristics may be associated with attitudes toward testing. Methods: Adult participants aged 18-85 years of age purchased a personalized genomic risk test at a subsidized rate and were administered a web-based health assessment that included questions regarding perceptions and attitudes toward undergoing testing. Results: Assessments were obtained for 3640 individual study participants, and $49.7 \%$ expressed overall concerns about undergoing testing. Logistic regression analysis revealed that women were more likely to express concerns (odds ratio $[\mathrm{OR}]=1.20,95 \%$ confidence interval [CI]: $1.04-1.39)$, as were individuals employed by a health care organization $(\mathrm{OR}=1.23,95 \% \mathrm{CI}: 1.04-1.46)$. Further, younger age $(\mathrm{OR}=0.97,95 \% \mathrm{CI}$ : $0.96-0.98)$, higher education $(\mathrm{OR}=$ $1.09,95 \%$ CI: $1.04-1.14)$, and higher trait anxiety $(\mathrm{OR}=1.28,95 \%$ CI: $1.20-1.37)$ were also significantly associated with expressing concerns related to testing. Attitudes regarding disclosure of genetic risk for a nonpreventable disease were also assessed. None of the individuals in our sample indicated that they would definitely not want to know their risk, and a total of $82.4 \%$ indicated that they would want to know. Conclusion: Among individuals who undergo direct-to-consumer genetic testing, approximately half still express concerns about the process/experience. Further, given that concerns vary among different subgroups of consumers, if the clinical validity and utility of these tests are demonstrated, tailored genetic education and counseling services may be of benefit. Genet Med 2010:12(9):556-566.
\end{abstract}

Key Words: direct-to-consumer, GWAS, genetic testing, personalized medicine, genomic risk assessment, disclosure of genetic risk

$\mathrm{O}$ ver the last few years, several genome-wide association studies (GWASs) have resulted in the discovery of specific genetic variations found to be associated with more than 80 common, polygenic diseases. ${ }^{1}$ Based on these findings and the increasing feasibility of performing genome-wide single-nucleotide polymorphism scans, a number of companies ${ }^{2-5}$ now offer tests that aim to calculate an individual's risk for these common diseases using this genome-wide technology. These companies

From the ${ }^{1}$ Scripps Genomic Medicine, Scripps Translational Science Institute, and Scripps Health, La Jolla, California; ${ }^{2}$ Scripps Clinic, La Jolla, California; ${ }^{3}$ Navigenics, Foster City, California; and ${ }^{4}$ Department of Molecular and Experimental Medicine, The Scripps Research Institute, La Jolla, California.

Eric J. Topol, MD, Scripps Translational Science Institute, 3344 N. Torrey Pines Court, Suite 300, La Jolla, CA 92037. E-mail: etopol@scripps.edu.

Disclosure: Drs. Cargill and Vanier and Ms. Silver are employees of Navigenics. The other authors declare no conflict of interest.

Supplemental digital content is available for this article. Direct URL citations appear in the printed text and are provided in the HTML and PDF versions of this article on the journal's Web site (www.geneticsinmedicine.org).

Submitted for publication January 14, 2010.

Accepted for publication June 2, 2010.

Published online ahead of print August 17, 2010.

DOI: $10.1097 /$ GIM.0b013e3181eb51c6 offer these tests, for a fee, direct-to-consumer (DTC) over the internet. The purchase of these tests is ultimately initiated by consumers without the obligatory involvement of the consumer's health care provider. ${ }^{6}$

The "personal genomics services" testing offered by these companies is distinguished from previous DTC genetic testing services in several ways. These tests examine, and disclose to consumers simultaneously, the results of high throughput genome-wide genotyping 6 and risk estimates for approximately 20-40 (depending on the company) common, polygenic diseases. In addition, the risk estimates provided are probabilistic rather than deterministic and are only associated with modest changes in risk. Although the analytic validity of these tests is good, serious questions have been raised regarding the consistency and accuracy, ${ }^{7}$ clinical validity, clinical utility, ${ }^{8}$ and actionability ${ }^{9}$ of DTC GWAS-based risk results. In addition, the risk estimates provided do not take other risk factors into account, such as family history. ${ }^{10}$

In addition to issues related to clinical validity and utility, there is also a lack of research on how best to present this type of risk information to individual consumers, ${ }^{11}$ and on how individual consumers are likely to respond to their results. It is an open question as to whether this information would lead consumers to (a) make positive health behavior changes in response to their results; (b) adopt more fatalistic attitudes and/or experience high levels of anxiety in response to estimates of high risk; or perhaps worse yet, (c) be falsely reassured by inaccurate estimates of low risk. This lack of research represents a gap that can potentially be filled by framing each of the pros and cons related to GWAS-based DTC genetic testing as research questions and testable hypotheses. ${ }^{11}$

To begin to bridge this gap, the Scripps Genomic Health Initiative (SGHI) was designed to assess consumer response to DTC personalized genomic risk assessment. SGHI participants purchased a GWAS-based DTC genetic test at a subsidized rate and were administered baseline (i.e., pre-risk disclosure) and 3-and 12-month follow-up web-based health assessments. The design of the SGHI was based on previous studies that have examined the behavioral and psychological impact of genetic risk disclosure for single diseases ${ }^{12}$ and as such, focuses on assessment of surveillance/health screening behaviors, lifestyle (i.e., diet and exercise), and psychological outcomes.

To our knowledge, there are at least two other large-scale studies investigating the behavioral and psychosocial impacts of GWAS-based DTC genetic testing. One is being spearheaded by the Coriell Institute (available at: www.coriell.org/index. $\mathrm{php} / \mathrm{content} / \mathrm{view} / 92 / 167 /$ ), and the other, termed the "Multiplex Initiative," is led by scientists at the National Human Genome Research Institute. ${ }^{11,13}$ Both studies have several notable strengths, including sample sizes in the thousands and, perhaps more critically, study designs that involve the in-depth assessment and tracking of individuals who opt not to be tested, which is not a feature of the SGHI. However, neither study includes evaluation of a currently marketed version of a GWAS-based 
DTC personalized genomic risk assessment product nor do they require a copayment, both of which are features of the SGHI. For this reason, the SGHI may be uniquely positioned to address research questions that more closely pertain to the "real world" interface between the GWAS-based DTC genetic testing industry and the public. However, we note that there are still some significant differences (e.g., copayment amount) between the uptake process for our study participants relative to the process for members of the general population who elect to undergo testing.

It is noteworthy that previous studies that have examined the behavioral and psychological impacts of genetic risk disclosure have been mostly small, in some cases underpowered, and thus not surprisingly, somewhat mixed in terms of their findings. ${ }^{12,14}$ In addition, issues related to consumer characteristics and the potential for the mode of delivery of these tests (e.g. via the internet and at least currently, at prices not affordable to many Americans) to increase existing health disparities ${ }^{15-17}$ have also been raised. Also, although a few studies have emerged that have explored the expectations, perceptions, and attitudes of "likely consumers,"18 as well as "users" of personalized genomic risk assessments, ${ }^{13}$ there is almost nothing known in this regard about individuals who actually go on to purchase a DTC GWAS-based risk assessment test. Thus, knowledge regarding consumer characteristics and perceptions of DTC personalized genomic risk assessment is needed. ${ }^{19}$

In this article, we explore the following aims: first, we describe the design and overall aims of the broader SGHI initiative, which are focused on assessing response to testing. Second, we leverage data from the Baseline Health Assessment to address several questions related to understanding the characteristics and attitudes of "early adopters" of GWAS-based DTC genetic testing. Third, we present some very basic and somewhat limited exploratory analyses comparing the characteristics of our sample to two groups of nonresponders in order to generate hypotheses regarding predictors of uptake. Fourth, we assess the extent to which study participants report concerns related to DTC personalized genomic risk assessment and evaluate factors associated with those concerns. Fifth, given previous evidence of demographic differences in attitudes toward genetic risk disclosure, in particular for nonpreventable diseases, ${ }^{20,21}$ we assessed this issue among our study participants within the context of GWAS-based DTC risk assessment.

\section{MATERIALS AND METHODS}

The study was approved by the Scripps Office for the Protection of Research Subjects (SOPRS) and institutional review boards (IRBs). Informed consent was obtained electronically from each study participant.

\section{Subjects}

\section{Study initiation}

In early 2008, scientists at the Scripps Translational Science Institute approached a number of the companies that were offering DTC personalized genomic risk assessment testing to discuss developing a collaboration in which their product would be offered to research participants. From this, an arrangement was made between Scripps Translational Science Institute and Navigenics to provide the Navigenics Health Compass to SGHI participants at a discounted rate.

\section{Recruitment}

The study was launched in October 2008, at which time recruitment efforts were targeted toward Scripps Health em- ployees, employee family members, and Scripps Health patients. A number of recruitment strategies were used, including all-employee "email blasts," announcements by weekly informational packets sent to managers, announcements at system wide, quarterly manager meetings, and postings on the Scripps employee intranet. As the study progressed, other employers were approached about the possibility of recruiting study participants from among their employees. Organizations in addition to Scripps Health from which study participants were eventually recruited included Microsoft, Sempra Energy, Qualcomm, SDG\&E, Life Technologies, Affymetrix, and The Scripps Research Institute.

Eligibility criteria were purposely broad to facilitate enrollment of as large and diverse a group of study participants as possible. These criteria included the following: (1) age 18 years or older; (2) valid e-mail address; and (3) ability to provide a copayment for the genomic risk assessment test.

\section{Procedures}

\section{Screening}

The SGHI used web-based informed consent and web-based health assessment questionnaires (see Fig. 1 for timeline and flowchart of study procedures). Interested individuals were directed to the Scripps landing page on the Navigenics website (available at: www.navigenics.com) where they could obtain information about the study, the personalized genomic risk assessment test, the IRB-approved Scripps informed consent, the Navigenics User Agreement, and e-mail contact information for Scripps study coordinators. Importantly, study participants were exposed to the same advertising claims and imagery used by Navigenics for recruitment of nonstudy participant consumers; layered on this, however, was the requirement for study participants to additionally review Scripps IRB documents and ultimately give their consent to participate. Once the informed consent was complete, participants were asked for their payment information, and directed to www.surveymonkey.com to complete the Baseline Health Assessment and provide a 2-mL

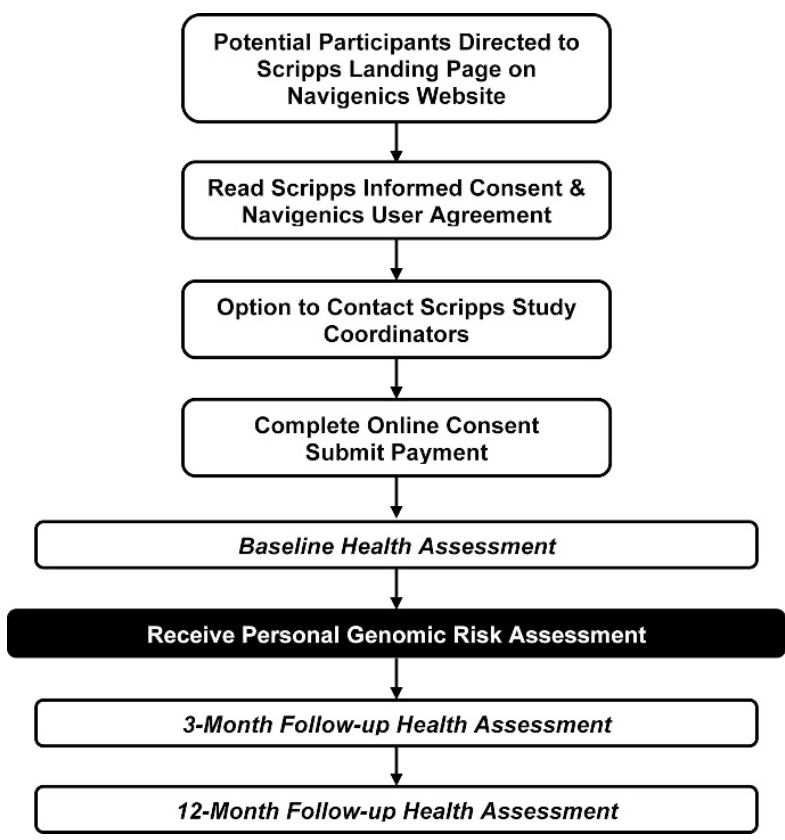

Fig. 1. Timeline and flowchart of study procedures. 
saliva sample. They could either have a saliva collection kit mailed to their home or they could choose to attend a scheduled "saliva collection event" on the Scripps campus where SGHI study coordinators provided assistance and answered questions.

\section{Copayment}

The initial cost to participate was $\$ 150$, and to encourage early enrollment, the study was designed such that the copayment increased over time; the highest amount, charged in the final months of the study, was $\$ 470$. In addition, for a small fraction of participants, the risk assessment was fully subsidized by their employer.

\section{SGHI assessments}

\section{Baseline health assessment overview}

All SGHI assessments were administered using the webbased survey tool, SurveyMonkey (available at: http://www. surveymonkey.com/). The Baseline Health Assessment included items that assessed personal demographics, family health-span history, personal/individual health-span history, behavioral health domains (see "Behavioral health measures"), items to characterize current health status (see "Health care status"), and questions pertaining to perceptions and attitudes toward DTC personalized genomic risk assessment testing (see "Perceptions of DTC genetic testing"). The survey instrument was pretested by 12 research staff members and revised accordingly before being administered to study participants. The distribution of completion times for the Baseline Health Assessment was found to be positively skewed, and the overall median time to complete was 33 minutes.

\section{Behavioral health measures}

The behavioral health component of the SGHI was focused on assessment of surveillance/health screening behaviors, lifestyle (i.e., diet and exercise), and psychological functioning (i.e., anxiety). The selection of behavioral assessment instruments to measure these domains was based on several criteria, including that each measure show evidence of reliability and validity, be brief, published, and require no more than an 8th grade reading level to complete. The primary instruments used in our Baseline Health Assessment, all of which were readministered at the three and 12-month follow-up assessments, were as follows: Surveillance/Health Screening Behaviors-California Health Interview Survey Adult Questionnaire, ${ }^{22}$ Diet-The Food Screener, ${ }^{23}$ Exercise - Godin Leisure-Time Exercise Questionnaire, ${ }^{24}$ and State Anxiety-Spielberger State-Trait Anxiety Inventory (STAI). ${ }^{25}$

Although the SGHI closed enrollment in September 2009, longitudinal follow-up data collection remains ongoing. Thus, this report does not include any analysis of data on response to testing; rather, it is focused on analysis of baseline data pertaining to study participant perceptions and attitudes toward testing.

\section{Health care status}

Three questions from the Baseline Health Assessment were used to measure access to health care and perceptions of current health status among our study participant sample. The first question read as follows: "How often do you visit your physician or health-care provider?" Response options are listed in Table 1 under "Physician Visit Frequency." The second question was "Do you have health insurance?" (yes/no). Finally, to get a sense of how study participants perceived their own current health status, we asked "How is your health?" Response
Table 1 Sample descriptive statistics for $N=3640$ study participants

\begin{tabular}{lc}
\hline $\begin{array}{l}\text { Demographics and health information } \\
\text { seeking factors }\end{array}$ & $\begin{array}{c}\text { Sample } \\
\text { characteristics }\end{array}$ \\
\hline Gender (\% female) & 53.0 \\
Age (mean, SD) & $46.2(11.8)$ \\
Household income (median category) & $100 \mathrm{k}-149 \mathrm{k}$ \\
Individual household income categories & \\
$\quad$ (percentage of sample) & \\
$50 \mathrm{k}$ & 6.6 \\
$50 \mathrm{k}-99 \mathrm{k}$ & 21.9 \\
$100 \mathrm{k}-149 \mathrm{k}$ & 24.5 \\
$150 \mathrm{k}-199 \mathrm{k}$ & 16.5 \\
$200 \mathrm{k}-249 \mathrm{k}$ & 9.6 \\
$250 \mathrm{k}-299 \mathrm{k}$ & 6.4 \\
$>300 \mathrm{k}$ & 14.6
\end{tabular}

Education (median category)

Some postcollege education

Individual education category (percentage of sample)

Under 11 yr

0.4

HS graduate or GED

10.1

2-year college graduate

10.6

4-year college graduate

27.0

Some postcollege education

14.5

Master's degree

22.1

Professional degree or $\mathrm{PhD}$

15.2

Ethnicity (\% white)

80.3

Health-related occupation (\% SH employee)

21.0

Trait Anxiety $Z$ score (mean, SD, median)

$-0.07(1.1),-0.23$

Frequency visit physician (median)

Once per year

Individual physician visit frequency category (percentage of sample)

Less than once every 2 years

Every 2 years

Once per year

Twice per year

20.4

Between 3 and 6 times per year

Between 6 and 10 times per year

More than 10 times per year

1.5

Perceived health status (mode)

Very good

Individual health status category (percentage of sample)

Very good

50.4

Good

Average

10.9

Poor

Very poor

0.1 
options are again listed in Table 1 under "Perceived Health Status."

\section{Perceptions of DTC genetic testing}

Two questions included on the Baseline Health Assessment pertaining to study participant perceptions and attitudes toward DTC personalized genomic risk assessment were analyzed. The first question aimed to measure study participant concerns about DTC personalized genomic risk assessment and read as follows: "Do you have concerns about participating in this initiative? Please check all that apply." Response options were "Concerns related to learning about my disease risk from my DNA testing," "Concerns related to not knowing how I will feel about my results," "Concerns related to the quality and reliability of the testing lab and the results," "Concerns related to potential privacy issues about my data," and "I do not have concerns about participating in this initiative." The second question aimed to measure study participant attitudes and desires regarding receipt of potentially distressing genetic test results and read as follows: "Would you want to know your genetic risk for a disease that you couldn't do anything to prevent? Please check all that apply." Response options were "Definitely no," "Possibly, if I would get the disease very far in the future," "Possibly, if it's a disease that's not fatal," "Possibly, if it would give me the opportunity to put my affairs in order (e.g., legal, financial)," "Possibly, if it would give me the opportunity to live my life differently (e.g., spend more time with family, travel)," "Yes," and "Other (please specify)."

\section{Data analysis}

All statistical analyses were conducted using the statistical software packages SPSS, R, and the Dimension Research Inc. resources for computing $Z$ tests and confidence intervals for proportions. ${ }^{26}$ Data were screened for the presence of extreme cases. Although age values were found to be generally normally distributed, trait anxiety $Z$ scores were positively skewed, and thus, analyses of this variable were conducted using nonparametric statistics. Analyses using the $Z$ test for two proportions were performed comparing study participants identified as (a) Scripps Health ( $\mathrm{SH})$ employees and study participants identified as (b) Microsoft employees to basic demographic data available for employees of each of these two companies overall. 27,28

Subsequent analyses were framed in terms of two primary dependent variables: (a) expressing overall concerns related to personal genomic risk assessment (yes/no) and (b) desire to know genetic risk for a nonpreventable disease (yes/possibly; this dichotomization was used because there were no individuals in the sample who responded "no" to this question). Secondary dependent variables included expressing specific concerns related to DTC genetic testing (listed above) and indicating specific scenarios in which one may want to know genetic risk for a disease that is not preventable (also listed above). Independent variables included a number of factors previously identified as predictors of health information seeking, ${ }^{29}$ including demographics, personal salience or involvement (i.e., being in a health-related occupation), psychological functioning (i.e., trait anxiety $Z$ score), and perceived information gathering capacity (i.e., access to health care as indexed by self-reported frequency of physician visits and perceived current health status). Year of enrollment in the study was subtracted from year of birth to determine approximate age. Study participants selected their household income category and their education category; response categories in both cases were ranked (1-7) based on increasing income and increasing education, respectively, and each individual's rank was used in subsequent analyses. Ethnicity was dichotomized as yes/no self-reported white. Being in a health-related occupation was operationally defined as whether the study participant was a $\mathrm{SH}$ employee. Trait-related anxiety was assessed by the trait anxiety subscale of the STAI. Cronbach $\alpha$ for the current sample was 0.932; raw scores were transformed into $Z$ scores based on age and gender normative data. ${ }^{25}$ Study participants selected their frequency of physician visits, and responses were ranked (1-7) based on increasing frequency.

In terms of bivariate associations, $\chi^{2}$ tests with one degree of freedom were used to examine the relationship between categorical independent variables and our primary and secondary dependent variables. Associations with income, education, trait anxiety $Z$ scores, and physician visit frequencies were assessed using Mann-Whitney $U$ tests. ${ }^{30}$ Associations with age were tested using an independent samples $t$ test. A logistic regression model was used to evaluate multivariate relationships and the extent to which each independent variable, in the presence of all the other independent variables, contributed uniquely to the prediction of each of our primary dependent variables. All reported $P$ values are uncorrected for multiple testing.

\section{RESULTS}

\section{Sample characteristics}

\section{Inclusions/exclusions}

A total of 4884 individuals enrolled in the study, meaning that they completed the informed consent and provided payment for the personalized genomic risk assessment test. For 736 of these individuals, because of an inadvertent protocol deviation, genetic test results were released before completion of the Baseline Health Assessment rendering these individuals ineligible for the remainder of the study. A total of 336 individuals were lost to follow-up because of either partial completion of the Baseline Health Assessment or noncompletion after three e-mail reminders. Baseline Health Assessments were completed for the remaining 3812 individuals; however, surveys were submitted twice by 106 individuals and three times by four individuals; thus, these individuals were excluded, leaving a remaining subject total of 3702 individuals. Of these, a total of 55 individuals canceled their order (most commonly cited reasons for this were financial reasons, insurance concerns, and change of mind), and after additional quality control analyses, it was also discovered that seven individuals completed the Baseline Health Assessment after their genetic test results were received. This left a total of 3640 study participants for whom a Baseline Health Assessment was available and could be analyzed in this study. In terms of assessment completion, this is consistent with a response rate of $74.5 \%$ and a cooperation rate of $87.1 \%$ as defined by the American Association for Public Opinion Research. ${ }^{31}$

\section{Descriptive statistics}

Sample descriptive statistics are presented in Table 1. With respect to the sample as a whole, ages ranged from 18 to 85 years. Trait anxiety $Z$ scores ranged from -1.70 to 5.30 . The distribution of individuals across the range of physician visit frequencies is shown in Table 1 . A total of $98.9 \%$ of the sample indicated that they had health insurance. The frequency of responses relating to self-reported current health status is also listed in Table 1. Given that nearly all study participants reported having health insurance, this variable was not included in further analyses. In addition, because the majority of individuals reported their perceived current health status to be "very good," 


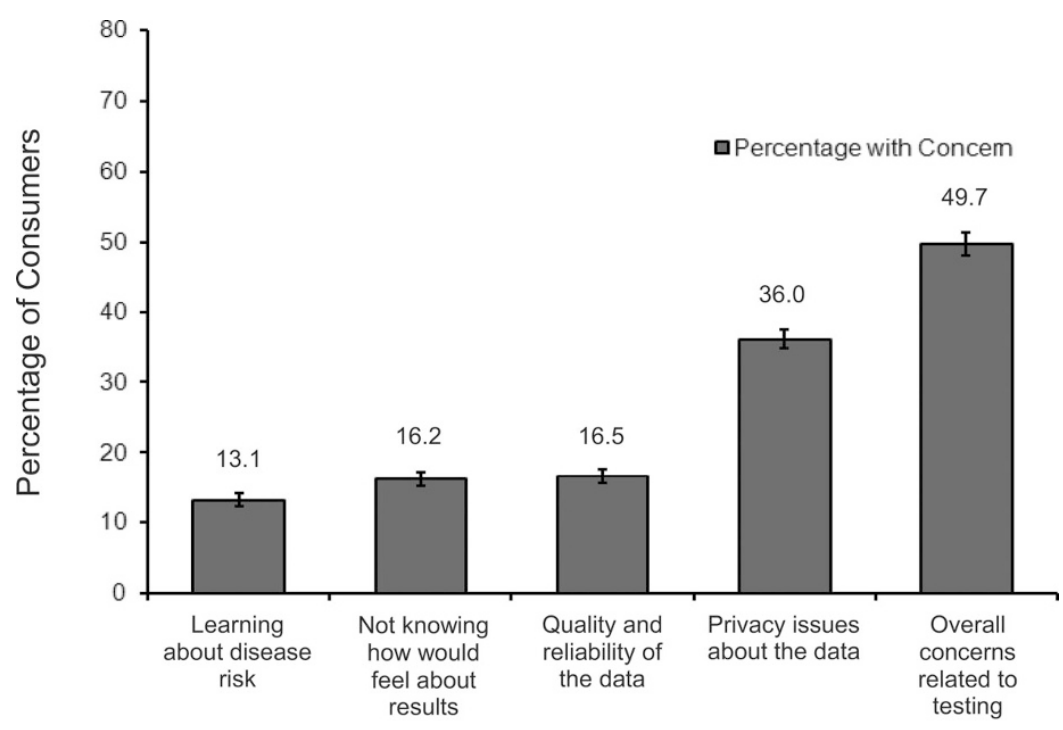

Concerns Regarding DTC Personalized

Genomic Risk Assessment

Fig. 2. Study participant concerns related to DTC personalized genomic risk assessment. Percentage of study participants reporting specific concerns related to DTC personalized genomic risk assessment despite ultimately choosing to participate in the study. Error bars represent the $95 \%$ confidence interval for the proportion. Note that respondents could select multiple specific concerns, thus percentage totals sum to $>100$.

this variable was dichotomized (i.e., "very good" yes/no) in subsequent analyses.

\section{Demographics of study participants versus nonresponders}

The gender and ethnic make-up of individuals from two specific recruitment sources (i.e., SH and Microsoft) were compared with the gender and ethnic make-up of the sampling frame of employees (i.e., total US employees) from each of these companies as a whole. Although the gender make-up of study participants was consistent with that of nonresponders, a significantly lower proportion of study participants were nonwhite relative to nonresponders. These results held for both $\mathrm{SH}$ (gender $Z=0.599, P>0.05$ and ethnicity $Z=15.481, P<$ 0.05 ) and Microsoft (gender $Z=0.985, P>0.05$ and ethnicity $Z=6.693, P<0.05)$.

\section{Concerns related to DTC genetic testing}

The percentages of study participants endorsing concerns related to DTC personalized genomic risk testing are shown in Fig. 2. A total of $49.7 \%$ indicated that they had overall concerns despite ultimately choosing to participate in the study. Among those expressing overall concerns, the specific concerns expressed are shown, with the most common being concerns related to privacy issues.

Bivariate associations with expressing overall concerns related to testing and each of our independent variables are shown in Table 2. Results indicated that the proportions of women $(P<0.001)$, individuals in a health-related occupation $(P<$ $0.01)$, and individuals who perceive their health to be less than "very good" $(P<0.01)$ were higher among those expressing overall concerns related to testing. Further, younger age $(t(3,638)=10.4, P<0.0001)$, lower income $(Z=-3.2, P<$ $0.01)$, and higher trait anxiety $(Z=-9.9, P<0.0001)$ were also associated with expressing concerns.
Results of the logistic regression model analysis to evaluate predictors of expressing concerns related to testing in a multivariate context are also presented in Table 2. In this analysis, gender, age, education, having a health-related occupation, and level of trait anxiety were all significant predictors of expressing overall concerns related to testing. The nature of the relationships between expressing overall concerns related to testing and age and trait anxiety, respectively, were further explored. As shown, the likelihood of expressing concerns related to testing shows a steady decrease with age (Fig. 3A) and a steady increase with increasing trait anxiety (Fig. 3B). In addition, individuals with lower education were less likely to express concerns (Fig., Supplemental Digital Content 1, http://links.lww.com/GIM/A114).

Bivariate associations with expressing specific concerns related to testing are shown in Table, Supplemental Digital Content 2, http://links.lww.com/GIM/A115.

\section{Knowledge of genetic risk for nonpreventable disease}

The percentages of study participants indicating whether they would want to know their genetic risk for a nonpreventable disease are shown in Fig. 4. Notably, no individuals in our sample indicated that they would definitely not want to know their risk, whereas a total of $82.4 \%$ indicated that they would want to know. Among those individuals indicating that they were unsure about whether they would want to know their risk, the specific scenarios in which they would consider receiving risk information are shown, with the most common one being if one could live life differently.

Bivariate associations with uncertainty regarding receipt of genetic risk information for a nonpreventable disease are shown in Table 2. Results show that proportions of women $(P<$ $0.001)$, individuals of self-reported white ethnicity $(P<0.05)$, and individuals in a health-related occupation $(P<0.01)$ were higher among study participants who indicated that they were 


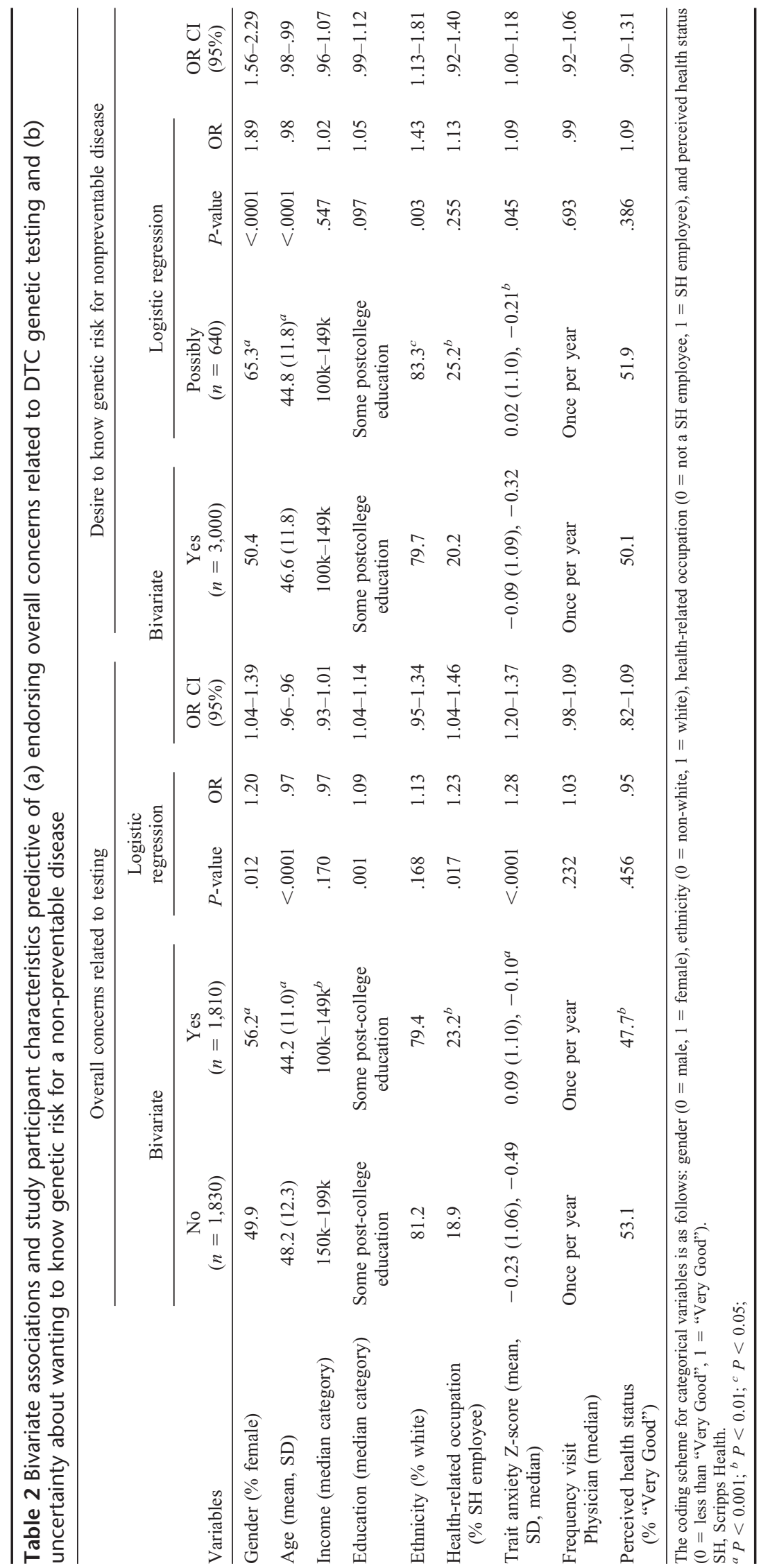



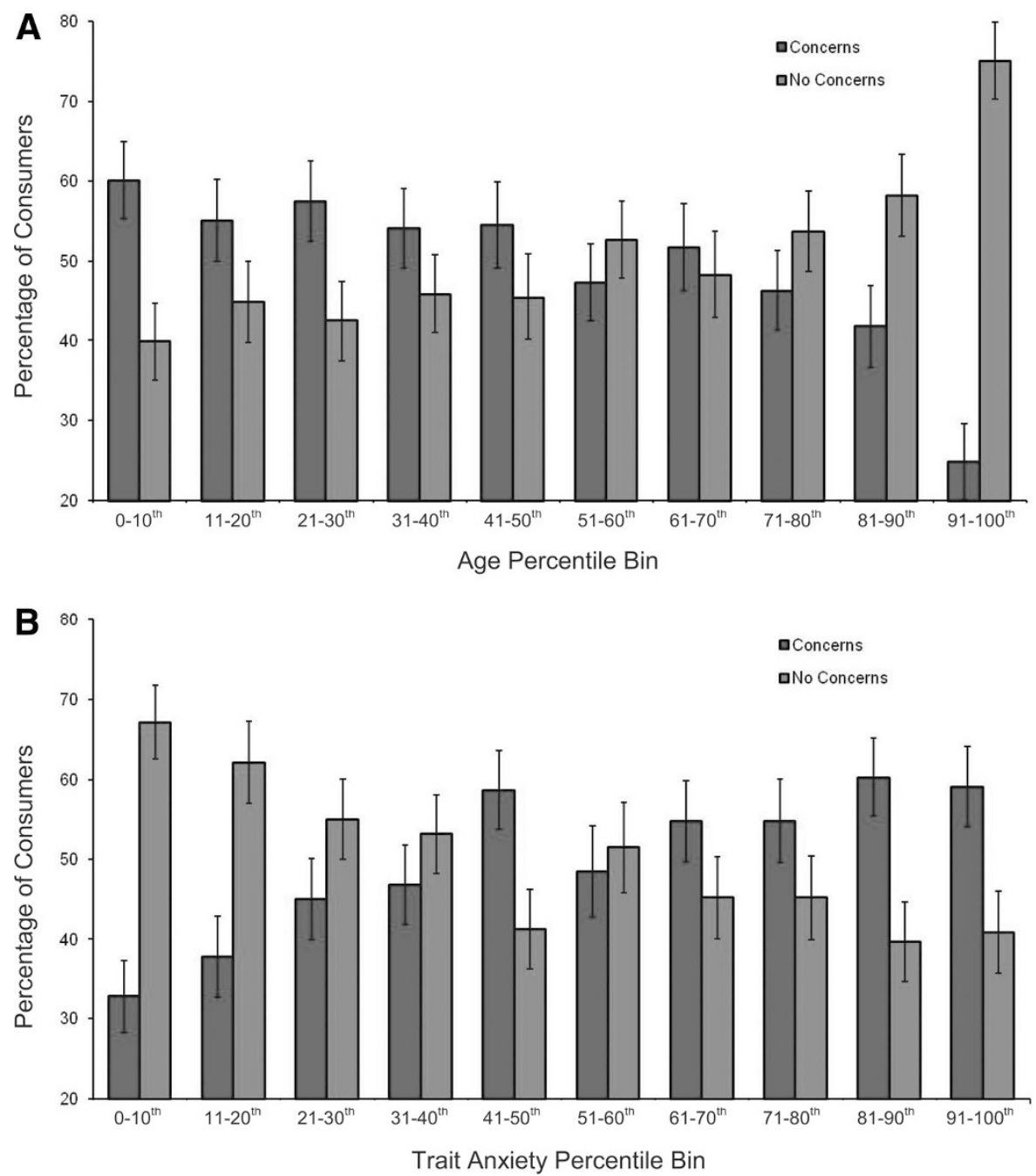

Fig. 3. Variability in overall concerns related to DTC genetic testing as a function of age and trait anxiety. A, Relationship between age and endorsing concerns related to DTC genetic testing. Younger individuals are more likely to endorse concerns related to testing. The age groups in years within each percentile bin are as follows: $0-10$ th (18-30); 11-20th (31-35); 21-30th (36-39); 31-40th (40-43); 41-50th (44-46); 51-60th (47-50); 61-70th (51-53); 71-80th (54-57); 81-90th (58-62); and 91-100th (63-85). Error bars represent the 95\% confidence interval for the proportion within each percentile bin. B, Relationship between trait anxiety and endorsing concerns related to DTC genetic testing. Individuals with higher trait anxiety are more likely to endorse concerns related to testing. Error bars represent the $95 \%$ confidence interval for the proportion within each percentile bin.

unsure about whether they would want to know their genetic risk for a nonpreventable disease. Further, younger age $(t(3,638)=-3.5, P<0.001)$ and higher trait anxiety $(Z=$ $-2.67, P<0.01)$ were also both associated with uncertainty regarding risk disclosure.

Results of the logistic regression model analysis for this dependent variable are shown in Table 2. In this multivariate context, gender, age (Fig. A, Supplemental Digital Content 3, http://links.lww.com/GIM/A116), ethnicity, and level of trait anxiety (Fig. B, Supplemental Digital Content 3, http://links.lww.com/GIM/A116) were all significant predictors of uncertainty regarding risk disclosure for a nonpreventable disease.

Bivariate associations with specific scenarios in which one may want to know genetic risk for a disease that is not preventable are shown in Table, Supplemental Digital Content 4, http://links.lww.com/GIM/A117.

\section{DISCUSSION}

In this report, we have described a large group of individuals predominately employed by health and technology companies who elected to purchase a DTC personalized genomic risk test when recruited as part of a study. Our sample reflects a wide age range of individuals who are highly educated, generally well-off financially, have adequate access to health care, and consider themselves to be in good health. Limited, exploratory analyses comparing the characteristics of our sample of study participants to two groups of nonresponders suggest that non-white individuals may be less likely to purchase and undergo DTC 


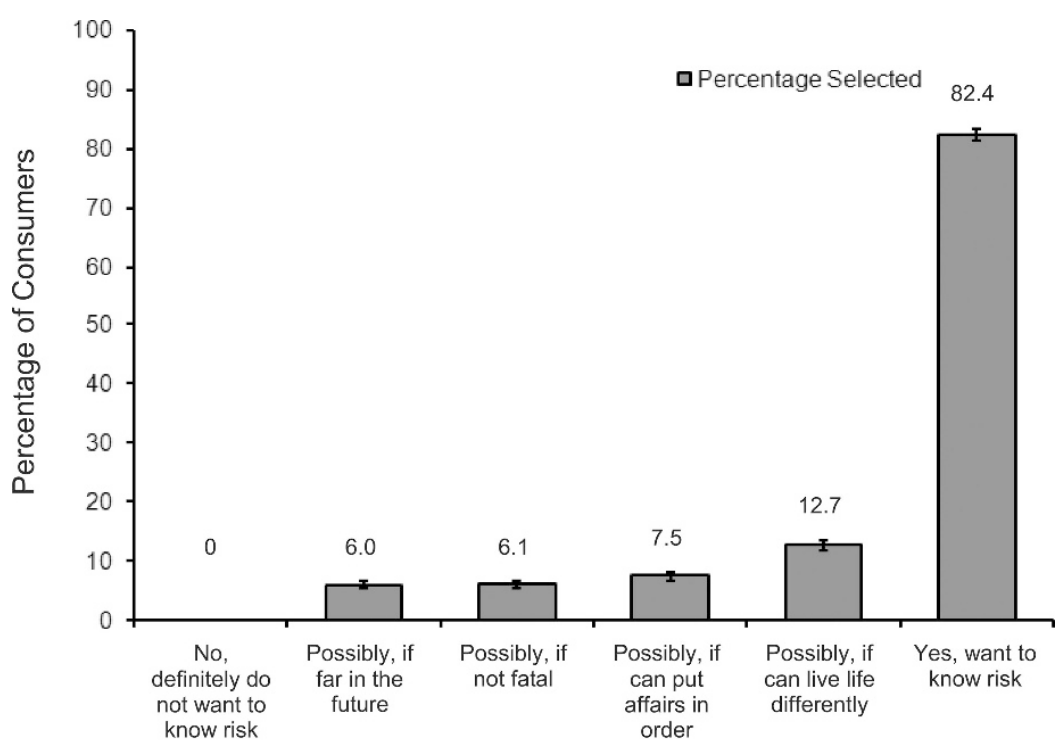

Responses Regarding Desire to Know

Genetic Risk for Non-Preventable Disease

Fig. 4. Responses regarding desire to know genetic risk for a disease that is not preventable. Study participants' attitudes regarding disclosure of genetic risk for a nonpreventable disease. Error bars represent the $95 \%$ confidence interval for the proportion. Note that respondents could select multiple specific scenarios, thus percentage totals sum to $>100$.

personalized genomic risk assessment. We also found that when asked, approximately half of our sample expressed overall concerns related to testing despite ultimately choosing to participate in the study. In addition, more than $80 \%$ of study participants indicated they would want to know their genetic risk even for a nonpreventable disease. To our knowledge, this represents the first large-scale study to emerge in the literature of individuals who have purchased (albeit at a discounted rate) a currently marketed GWAS-based personalized genomic risk assessment product.

We compared sample demographics and significant associations from this study to other studies exploring interest in, opinions about, and use of genetic testing. Specifically, McGuire et al. ${ }^{18}$ recently studied social networkers' interest in and attitudes toward personalized genomic risk tests, arguing that because DTC testing is primarily offered through the internet, social networkers are likely to be early adopters of these services. Although there were few differences between the demographic characteristics of those who reported that they either "did use" or "would use" DTC genetic tests versus those who "would not use" them, the sample as a whole was predominately female, white, and highly educated; the vast majority $(85 \%)$ also reported having health insurance and a regular physician $(78 \%)$. Another study that surveyed awareness and use of DTC nutrigenomic tests ${ }^{15}$ compared those who reported being aware of DTC nutrigenomic tests versus those who did not and found that the former group tended to be younger, more highly educated, and have a higher income. Further, when actual users of DTC nutrigenomic tests (i.e., only $n=29$ of 5250 respondents total) were compared with those who were not aware of the tests, they found that users were also more likely to be female. In general, these findings are quite consistent with those from this study.

Our finding suggesting that white individuals may be more likely to undergo DTC risk testing relative to non-white indi- viduals is also consistent with previous findings of racial and ethnic disparities in access to and utilization of a number of health care services, ${ }^{16}$ including uptake of genetic testing for single diseases such as breast cancer. ${ }^{17}$ Because we did not, however, examine differences in salary or health status between ethnic groups in our analysis (factors which could certainly be confounding this result), we stress that this result is preliminary and merely suggestive of an important area for further study.

In terms of perceptions of and attitudes toward testing, we found that when asked, $49.7 \%$ of our sample expressed concerns about undergoing DTC personalized genomic risk assessment and participating in the study, with smaller subgroups of the sample reporting more specific concerns (e.g., related to privacy). Although this implies that more than half of the study participants do not have concerns about undergoing testing, SGHI participants represent a "best-case scenario" because we are only surveying individuals who eventually underwent testing. Thus, the attitudes of nonparticipants and of the general population are likely to reflect greater levels of concern and/or suspicion (and lack of awareness) regarding DTC GWAS-based genetic testing, and data to support this assertion are starting to emerge. ${ }^{19}$ This suggests that if the clinical validity and utility of GWAS-based risk assessments are demonstrated, community education plans that provide information regarding the intent and the possible beneficial outcomes of genomic medicine for the individual, ${ }^{19}$ if any, may be warranted. Notably, these results also indicate that even though a person undergoes DTC genetic testing, that person may still have any one of a number of concerns about the process/experience.

We found that the likelihood of expressing concerns decreased with advancing age and decreasing levels of trait anxiety. A possible explanation for this age pattern could be that the more years a person has lived, the more one already knows about their health and the diseases they will develop; thus, older individuals are less likely to receive risk information that is 
surprising or that may seem ominous. In regard to the trait anxiety finding, it is fairly intuitive that the more anxious one is by nature, the more one will experience general worry, including worry related to genetic testing. Although these findings are interesting, they are not necessarily surprising. Also, our large sample size contributes to the statistical significance of these predictors. We note that effect sizes are modest raising the issue of questionable clinical significance. Our finding that individuals with higher levels of education and those employed by a health care organization are also more likely to express concerns is similarly intuitive. Such individuals may be more aware of potential privacy risks, less trusting of entities associated with genetic testing, and in the case of health care employees, more aware of "weaknesses" in the system that may impact the individual.

Finally, no individuals in our sample indicated that they would definitely not want to know their risk for a nonpreventable disease, and a total of $82.4 \%$ indicated that they would want to know with remaining individuals expressing uncertainty in this regard. Although this question was admittedly abstract and did not provide respondents with a specific disease or set of symptoms to consider, it was still interesting to us that by far the largest effect size observed in this analysis was the extent to which women were overrepresented among those reporting uncertainty about wanting to receive risk results. According to a review by Marteau et al., ${ }^{20}$ women reportedly associate greater risks with technology than do males, even after accounting for differences related to knowledge about the risk itself. Similarly, others have further argued that genetic testing (at least traditionally) has a greater potential impact and relevance for women because of more salient concerns regarding reproduction. ${ }^{32}$ These arguments, however, contradict other observations concerning the role of gender and genetic testing and risk disclosure. For instance, a number of studies have found that women are more likely (not less) to request and undergo predictive genetic testing for Huntington disease, one salient example of a "nonpreventable" disease. ${ }^{33}$

In summary, this study has a number of implications. Our findings imply that uptake of DTC genetic testing may not be uniform across racial and ethnic groups suggesting that this may be an important area to monitor in terms of the potential for exacerbation of existing health disparities. Our results also suggest that even though a person undergoes DTC GWASbased genetic testing, that person may still have any one of a number of concerns about the process/experience. One interpretation of this might be that consumers may benefit from the provision of routine genetic counseling services for all consumers of DTC genetic testing products unless the individual explicitly "opts out." Finally, the finding that certain subgroups of consumers may have different perceptions/concerns regarding genomic risk assessment suggests that if the clinical validity/ utility of DTC GWAS-based genetic tests is demonstrated, consumers' needs in terms of genetic counseling services may vary as a function of demographic and other characteristics.

\section{Limitations}

Although the SGHI has a number of strengths, there are also some limitations regarding aspects of the study that should be stressed. First, we studied a sample of convenience with an unknown denominator; thus, strong conclusions regarding population-based rates of "uptake" are not warranted based on our data alone. Indeed, recent findings from another large scale study that closely tracks nonresponders suggest that when offered free genetic testing, less than one sixth of individuals may decide to be tested. ${ }^{13}$ Second, although part of the aim of the study was to evaluate attitudes and perceptions among "actual consumers" of a currently marketed GWAS-based risk test, we note that our sample is also not precisely representative of this broader population of consumers. The reasons for this are that (a) the study population was drawn largely from employees of a health care system and a number of technology and biotechnology companies - recruitment sources that are not representative of the general public; (b) our sample was recruited and did not seek out testing of their own desire or volition; and (c) participants were offered testing at a significant discount compared with what individuals in the general public would have to pay. Third, because we only surveyed individuals who agreed to purchase and undergo GWAS-based risk assessment, our data and conclusions are limited due to the absence of a control group. For example, gender, age and other differences seen in the number and types of concerns may not be representative of overall concerns with this type of testing; rather, we may simply be identifying individuals who are willing to undergo testing despite minor concerns. Along these same lines, our sample was also quite homogeneous, which similarly suggests that demographic differences between those that reported "any" versus "no" concerns may not be representative of the overall population.

We additionally note that our nonresponder comparison analysis of participants recruited from Microsoft was limited due to the fact that our Microsoft participants were not recruited from among all employees (the nonresponder comparison group) but rather only from one division of the company for which we were not able to obtain demographic descriptive data. Importantly, our recruitment strategy also allowed enrollment of spouses and family members. Given our study design, these relationships would be hard to discern with absolute certainty unless in obvious instances based on genetic data. Thus, in the context of this initial study, our analyses do not reflect exclusions for relatedness or household sharing, and therefore, to some extent, there may be observations that are nonindependent. Finally, the SGHI relies on web-based, self-report assessment of health behaviors. Although there is ample precedent for taking this approach ${ }^{34}$ and it allowed us to enroll a large number of individuals, self-report, in particular, is known to be less reliable than in-person clinical assessment.

\section{Future directions}

There is substantial controversy regarding the value of DTC personalized genetic testing, coupled with little available information pertaining to the impact of GWAS-based risk disclosure on consumers. This deficiency exists despite the fact that these tests are currently being marketed to the public and purchased by consumers, albeit on a limited basis. Empirical studies are needed to address these issues, which bear on important questions related to public health, the extent to which the DTC genetic testing industry requires additional oversight, and the future of personalized medicine.

Specifically, there are a number of questions for further study that have been raised by the current results. In regard to uptake of DTC genetic testing, controlled studies of population-based samples are required to determine whether the decision to undergo testing varies as a function of racial or ethnic group, and if so, whether this contributes to existing health disparities. In addition, in-depth, qualitative interview-based studies may be warranted to further understand consumers' attitudes toward testing, the nature of their specific concerns, and how these concerns may change with age/time, disease onset, the specific disease involved, and other familial, interpersonal, and social factors. 
More broadly, although there is a literature on the behavioral and psychological impact of risk disclosure for some select diseases, studies are needed that examine this impact in the context of risk disclosure for multiple common diseases/traits simultaneously. ${ }^{35}$ This is clearly a complicated research question, and creative study designs (e.g., constructing and analyzing composite risk scores and querying the participant regarding which risk estimates/conditions he or she is most and least concerned about) will be required. Another critical issue, previously suggested by others, ${ }^{36}$ is the extent to which recipients of GWAS-based risk information understand their results, including the probabilistic nature of the risk estimates provided. Research that evaluates the interplay between recipient "health literacy" and response to risk disclosure is needed. ${ }^{36}$ Along these same lines is the need to evaluate recipients' understanding of GWAS-based risk estimates in the context of other risk factors such as a positive family history for disease.

Most genetic risk disclosure research has shown that if they occur, negative psychological outcomes are temporary; however, further study of this in the context of GWAS-based risk disclosure is important, in particular given that results are most often provided directly to the consumer without an intermediary. Similarly, assessment of the behavioral impact, if any, is also critical, including the extent to which there are additional factors that may moderate this impact. For instance, are consumers who seek out genetic counseling when provided their results more likely to make positive behavioral changes (e.g., improving diet)? Others have suggested that the role of family and social networks may be important, ${ }^{36}$ and the question of whether positive behavioral change is more likely when multiple individuals in a family or household undergo testing. It may even be the case that additional genetic factors influence the extent to which individuals are able to make positive behavioral changes (e.g., genetic variants associated with satiety ${ }^{37}$ may influence adherence to diet recommendations). ${ }^{36}$ In the context of the SGHI, we have attempted to include assessment items and study design features that will allow us to address some of these research questions in the near future.

\section{CONCLUSION}

Many have made compelling arguments for increased regulation of the DTC personal genomics industry. It has also been noted, however, that too much regulation may limit future, and as yet unforeseen, benefits associated with DTC GWAS-based genetic testing and that society might actually be better served with minimal regulation to allow users in the marketplace to experience its benefits and fine tune its weaknesses. ${ }^{38}$ Empirical studies, such as the SGHI, should shed light on some of these issues. For now, the relatively easy availability of DTC genetic testing, cost notwithstanding, has led us into a new era characterized by what has been termed the "democratization of genomics" and genomic knowledge. ${ }^{39}$ As the cost of genotyping - and in the future, whole-genome sequencing-continues to drop, the number of individuals who seek personal genomic information and undergo testing may increase, possibly ushering in a health care system that is more consumer driven. The extent to which this is beneficial, particularly in regard to GWAS-based DTC genetic tests, will critically depend on efforts to demonstrate their clinical validity and utility. ${ }^{9}$ In the event that validity and utility is confirmed, this will further depend on efforts to educate physicians and other health care providers $^{40}$ and initiatives to increase public education and evaluate health literacy regarding genomic medicine.

\section{ACKNOWLEDGMENTS}

This work was supported in part by a NIH/NCRR flagship Clinical and Translational Science Award grant (1U54RR025204-01; PI: Eric J. Topol, MD) and Scripps Genomic Medicine Division of Scripps Health.

\section{REFERENCES}

1. Manolio TA, Brooks LD, Collins FS. A HapMap harvest of insights into the genetics of common disease. J Clin Invest 2008;118:1590-1605.

2. Available at: http://www.navigenics.com/. Accessed June 4, 2009

3. Available at: http://www.decodeme.com/. Accessed June 4, 2009.

4. Available at: https://www.23andme.com/. Accessed June 4, 2009.

5. Available at: http://www.pathway.com/. Accessed January 13, 2010.

6. Gurwitz D, Bregman-Eschet Y. Personal genomics services: whose genomes? Eur J Hum Genet 2009;17:883-889.

7. Ng PC, Murray SS, Levy S, Venter JC. An agenda for personalized medicine. Nature 2009;461:724-726.

8. Janssens AC, Gwinn M, Bradley LA, Oostra BA, van Duijn CM, Khoury MJ. A critical appraisal of the scientific basis of commercial genomic profiles used to assess health risks and personalize health interventions. Am J Hum Genet 2008;82:593-599.

9. Eng C, Sharp RR. Bioethical and clinical dilemmas of direct-to-consumer personal genomic testing: the problem of misattributed equivalence. $\mathrm{Sci}$ Transl Med 2010;2:17 cm15:1-5.

10. Valdez R, Yoon PW, Qureshi N, Green RF, Khoury MJ. Family history in public health practice: a genomic tool for disease prevention and health promotion. Annu Rev Public Health 2010;31:69-87 1 p following 87.

11. McBride CM, Alford SH, Reid RJ, Larson EB, Baxevanis AD, Brody LC. Putting science over supposition in the arena of personalized genomics. Nat Genet 2008;40:939-942.

12. Heshka JT, Palleschi C, Howley H, Wilson B, Wells PS. A systematic review of perceived risks, psychological and behavioral impacts of genetic testing. Genet Med 2008;10:19-32.

13. McBride CM, Alford SH, Reid RJ, Larson EB, Baxevanis AD, Brody LC. Characteristics of users of online personalized genomic risk assessments: implications for physician-patient interactions. Genet Med 2009;11:582587.

14. Botkin JR, Smith KR, Croyle RT, et al. Genetic testing for a BRCA1 mutation: prophylactic surgery and screening behavior in women 2 years post testing. Am J Med Genet A 2003;118A:201-209.

15. Goddard KA, Moore C, Ottman D, Szegda KL, Bradley L, Khoury MJ. Awareness and use of direct-to-consumer nutrigenomic tests, United States, 2006. Genet Med 2007;9:510-517.

16. Suther S, Kiros GE. Barriers to the use of genetic testing: a study of racial and ethnic disparities. Genet Med 2009;11:655-662.

17. Armstrong K, Micco E, Carney A, Stopfer J, Putt M. Racial differences in the use of BRCA1/2 testing among women with a family history of breast or ovarian cancer. JAMA 2005;293:1729-1736.

18. McGuire AL, Diaz CM, Wang T, Hilsenbeck SG. Social networkers' attitudes toward direct-to-consumer personal genome testing. Am J Bioeth 2009;9:3-10

19. Hahn S, Letvak S, Powell K, et al. A community's awareness and perceptions of genomic medicine. Public Health Genomics 2010;13:63-71.

20. Marteau TM, Dundas R, Axworthy D. Long-term cognitive and emotional impact of genetic testing for carriers of cystic fibrosis: the effects of test result and gender. Health Psychol 1997;16:51-62.

21. Taylor S. Gender differences in attitudes among those at risk for Huntington's disease. Genet Test 2005;9:152-157.

22. CHIS. California Health Interview Survey. Los Angeles, CA: UCLA Center for Health Policy Research, 2007

23. Block G, Gillespie C, Rosenbaum EH, Jenson C. A rapid food screener to assess fat and fruit and vegetable intake. Am J Prev Med 2000;18:284-288.

24. Godin G, Shephard RJ. A simple method to assess exercise behavior in the community. Can J Appl Sport Sci 1985;10:141-146.

25. Spielberger CD, Gorsuch RL, Lushene PR, Vagg PR, Jacobs AG. Manual for the State-Trait Anxiety Inventory (Form Y). Palo Alto: Consulting Psychologists Press, Incorporated, 1983.

26. Available at: http://www.dimensionresearch.com/resources/resources overview.html. Accessed January 1, 2010

27. Available at: http://money.cnn.com/magazines/fortune/bestcompanies/2009/ snapshots/38.html. Accessed January 1, 2010 
28. Available at: http://money.cnn.com/magazines/fortune/bestcompanies/2009/ snapshots/59.html. Accessed January 1, 2010.

29. Griffin RJ, Dunwoody S, Neuwirth K. Proposed model of the relationship of risk information seeking and processing to the development of preventive behaviors. Environ Res 1999;80:S230-S245.

30. Heiman GW. Basic statistics for the behavioral sciences, 2nd ed. Boston, MA: Houghton Mifflin Co., 1996.

31. Available at: http://www.aapor.org/Standard_Definitions/1818.htm. Accessed December 31, 2010.

32. Shiloh S, Saxe L. Perceptions of risk in genetic counseling. Psychol Health 1989;3:45-61.

33. Hayden MR. Predictive testing for Huntington's disease: the calm after the storm. Lancet 2000;356:1944-1945.

34. Brigham J, Lessov-Schlaggar CN, Javitz HS, Krasnow RE, McElroy M, Swan GE. Test-retest reliability of web-based retrospective self-report of tobacco exposure and risk. J Med Internet Res 2009;11:e35.
35. Green RC, Roberts JS, Cupples LA, et al. Disclosure of APOE genotype for risk of Alzheimer's disease. N Engl J Med 2009;361:245-254.

36. McBride CM, Koehly LM, Sanderson SC, Kaphingst KA. The behavioral response to personalized genetic information: will genetic risk profiles motivate individuals and families to choose more healthful behaviors? Annu Rev Public Health 2010;31:89-103.

37. Rosado EL, Bressan J, Martins MF, Cecon PR, Martinez JA. Polymorphism in the PPARgamma2 and beta2-adrenergic genes and diet lipid effects on body composition, energy expenditure and eating behavior of obese women. Appetite 2007;49:635-643.

38. Ball E. Duke bioethicist reflects on personal genomics. Environmental Factor, March 2009.

39. Guthrie JJ. Craig Venter sees change from genomics. San Francisco Chronicle, June 11, 2009.

40. Healy M. As genetic testing races ahead, doctors are left behind. Los Angeles Times, October 24, 2009.

\section{Erratum}

Glycogen storage disease type III diagnosis and management guidelines: Erratum

In the article appearing on page 446 of volume 12, issue 7, several references in the text were incorrect. All instances of reference 22 cited in the text actually refer to reference 24 in the reference list, in text citations of reference 23 refer to 25 in the reference list, in text citations of reference 24 refer to 26 in the reference list, in text citations of reference 25 refer to 27 in the reference list, in text citations of reference 26 refer to 28 in the reference list, in text citations of reference 27 refer to 29 in the reference list, in text citations of reference 28 refer to 22 in the reference list, and in text citations of reference 29 refer to 23 in the reference list.

On page 452, in the Atherosclerosis section of this article, the reference for the report Hershkovitz et al. should be reference 82, not reference 81. On page 456, in the Musculoskeletal/Functional/Rehabilition, Neuromuscular section, the following sentence should end with references 49 and 100 from the reference list: "Individuals may have symmetrical, predominantly proximal or more generalized weakness, occasionally in association with hypertrophy/pseudohypertrophy of some muscles." ${ }^{, 9,100}$ The final error to note is that reference 102 is included in the reference list, but is not cited in the text.

These errors have been noted in the online version of the article, which is available at www.geneticsinmedicine.org.

\section{REFERENCE}

Kishnani PS, Austin SL, Arn P, Bali DS, Boney A, Case LE, et al. Glycogen storage disease type III diagnosis and management guidelines. Genet Med 2010;12;446-463. 